\title{
Quantitative CT Indexes and CT Visual Score in Interstitial Lung Abnormality as Indicators of Concurrent Lung Cancer
}

\author{
Akihiro Hotta ${ }^{*}$, Kazuhiro Suzuki', Mariko Fukui², Katsutoshi Ando ${ }^{3}$, \\ Kenji Suzuki², Ryohei Kuwatsuru1, Kazuya Takamochi² \\ ${ }^{1}$ Departments of Radiology, Faculty of Medicine, Juntendo University, Tokyo, Japan \\ ${ }^{2}$ Departments of Thoracic Surgery, Faculty of Medicine, Juntendo University, Tokyo, Japan \\ ${ }^{3}$ Departments of Respiratory Medicine, Faculty of Medicine, Juntendo University, Tokyo, Japan \\ Email: *ahotta@juntendo.ac.jp
}

How to cite this paper: Hotta, A., Suzuki, K., Fukui, M., Ando, K., Suzuki, K., Kuwatsuru, R. and Takamochi, K. (2019) Quantitative CT Indexes and CT Visual Score in Interstitial Lung Abnormality as Indicators of Concurrent Lung Cancer. Open Journal of Radiology, 9, 105-114. https://doi.org/10.4236/ojrad.2019.92010

Received: April 11, 2019

Accepted: May 3, 2019

Published: May 6, 2019

Copyright $\odot 2019$ by author(s) and Scientific Research Publishing Inc. This work is licensed under the Creative Commons Attribution International License (CC BY 4.0).

http://creativecommons.org/licenses/by/4.0/

\begin{abstract}
Background: The associations between the severity of interstitial lung disease and the stage and histologic type of concurrent lung cancer remain unknown. Purpose: To evaluate whether the severity of interstitial lung abnormality (ILA), as indicated by quantitative computed tomographic (CT) indexes and CT visual score, was correlated with the stage and histological type of concurrent lung cancer. Materials and Methods: Twenty-eight patients with surgically diagnosed lung cancer and ILA on CT were enrolled in this retrospective study. The subjects were allocated to one of three groups by histological type: adenocarcinoma group (13 subjects); squamous cell carcinoma group (10 subjects); and the other histological diagnosis group (5 subjects). Two independent observers evaluated the CT findings to determine the CT visual score, and the kurtosis and skewness of CT-based density histograms were determined. The relationships between severity of ILA and the pathological stage and histological type of concurrent lung cancer were evaluated. Results: There were no significant differences in the CT visual scores and quantitative indexes among the three groups. CT visual score was significantly negatively correlated with pathological stage $(r=-0.43, P=0.025)$. Conclusion: Patients can have only mild ILA on visual scoring but advanced lung cancer. Therefore, the frequency of follow-up examination should not be based on the severity of ILA on CT.
\end{abstract}

\section{Keywords}

CT Visual Score, Interstitial Lung Disease, Lung Cancer, Quantitative CT Index 


\section{Introduction}

The frequency of lung cancer is higher in patients with interstitial lung disease (ILD) than in the general population [1] [2] [3]. Several imaging studies of lung-cancer-associated ILD [4] [5] [6] have been reported: computed tomographic (CT) scans show ill-defined lesions mimicking air-space consolidation [4] or nodular lesions with sharp margins [5] [6], with most tumors located in the periphery of fibrotic lesions [4] [5] [6]. However, the associations between the severity of ILD and the stage and histologic type of concurrent lung cancer remain unknown.

There are several ways to evaluate the severity of ILD on CT images. Quantitative CT indexes and CT visual scores are known indicators of the severity of ILD [7] [8] [9] [10]. Computer-derived indexes, such as kurtosis and skewness, can be obtained from frequency histograms of thin-section CT scans of the lung. Kurtosis describes the sharpness of the peak in a histogram. A histogram that is more peaked than the normal distribution has a positive kurtosis value. Skewness refers to the asymmetry of a histogram. A histogram with a long tail to the right has a positive skewness value. Histograms from patients with ILD are less skewed and less kurtotic than those from healthy subjects. This is thought to result from an increase in soft tissue and decrease in air space [7]. Kurtosis and skewness are moderately correlated with the results of pulmonary function tests (forced vital capacity, forced expiratory volume in $1 \mathrm{~s}$, total lung capacity, and diffusing lung capacity) [7] [8]. Furthermore, kurtosis is an independent predictor of mortality [9]. CT visual score, which is a semi-quantitative score of the visual extent of interstitial change on CT images, is also useful for predicting clinical mortality [9] and outcomes [10].

We hypothesized that, if there were any relevance of these quantitative indexes to concurrent lung cancer, they could be used to help identify those ILD patients who were most at risk of lung cancer. Here, we evaluated radiographic interstitial lung abnormality (ILA) on CT of patients with lung cancer and investigated the relationship between quantitative indexes and visual scores of ILA and the stage or histologic type of concurrent lung cancer. Our aim was to determine whether there was any index that could be used to identify a group of ILA patients at high risk of lung cancer.

\section{Materials and Methods}

\subsection{Study Subjects}

The retrospective study population comprised patients with lung cancer that was surgically diagnosed at Juntendo University Hospital between November 2012 and February 2015. The study was approved by the ethics committee of our institute (approval No. 2015015). We reviewed the findings of those lung cancer patients who had undergone thin-section (2-mm slice thickness at 2-mm intervals) $\mathrm{CT}$ of the whole lungs in the period 2 months before surgery, and those patients with ILA on CT were enrolled in the study. All CT findings were reviewed 
anew by the authors (K.S., thoracic radiologist with 15 years' experience; K.A., pulmonologist with 7 years' experience; M.F., thoracic surgeon with 8 years' experience; and K.T., thoracic surgeon with 21 years' experience). ILA was diagnosed by CT on the basis of reported criteria [11] [12] [13] and by consensus of the authors. ILA was defined as follows: non-dependent ground glass attenuation, non-dependent reticular abnormality, traction bronchiectasis and honeycombing. Patients who had received radiation or chemotherapy before surgery were excluded, and finally 28 patients with ILA were enrolled. Twenty-one patients were male and seven were female. The age range was 55 to 85 years (mean \pm SD, 71 \pm 7 years). Twenty-four patients were smokers (ex-smoker or current smoker).

\subsection{CT Scan Protocol}

All patients underwent thin-section CT with a 16-detector-row CT scanner or 64-detector-row CT scanner (Aquilion 16 or Aquilion 64; Canon Medical Systems, Tochigi, Japan) using 1-mm collimators and scanning parameters of 120 $\mathrm{kVp}$ with automatic exposure. A magnified view of each lung was reconstructed by using the lung algorithm (2-mm thickness at 2-mm intervals; entire lungs).

\subsection{Histopathological Evaluation for Lung Cancer}

Data on pathological stage and histologic type of lung cancer were obtained from the postoperative histopathological diagnosis. Staging was performed according to the $7^{\text {th }}$ Edition TNM lung cancer staging system of the International Association for the Study of Lung Cancer (IASLC) Staging Committee. To determine the relationships between histologic type and severity of ILA, we categorized patients into three pathological groups: adenocarcinoma (AC), squamous cell carcinoma (SCC), and other (OT).

\subsection{CT Visual Scores}

The thin-section CT findings were graded on a scale of 1 through 5 (from least severe to most severe) on the basis of the following classification system: 1 , normal attenuation; 2, ground glass attenuation; 3 , reticular abnormality; 4, traction bronchiectasis; and 5 , honeycombing. This grading scale and the zones assessed were based on previous reports, with minor changes [10] [14]. The presence of each of the above-mentioned imaging features was assessed independently in each of three (upper, middle, and lower) zones of both lungs. The upper lung zone was defined as the area of lung above the level of the tracheal carina. The lower lung zone was defined as the area of lung below the level of the inferior pulmonary vein, and the middle lung zone was defined as the area of lung between the upper and lower zones. We estimated (to the nearest 10\%) the proportion of lung parenchyma displaying each of the five described imaging features. The abnormality score of each zone was calculated by multiplying the percentage area by the grading scale score ( 1 to 5 ). The three zone scores of both lungs were averaged to determine the total score for each patient (see example in Figure 
1(a), Figure 2(a)). Two radiologists independently evaluated all images.

\subsection{Quantitative CT Indexes}

Lung-associated data were extracted and the bronchi, vessels, and tumors were excluded automatically by using a commercially available image analysis system (Synapse Vincent; Fujifilm Medical, Tokyo, Japan). A frequency histogram

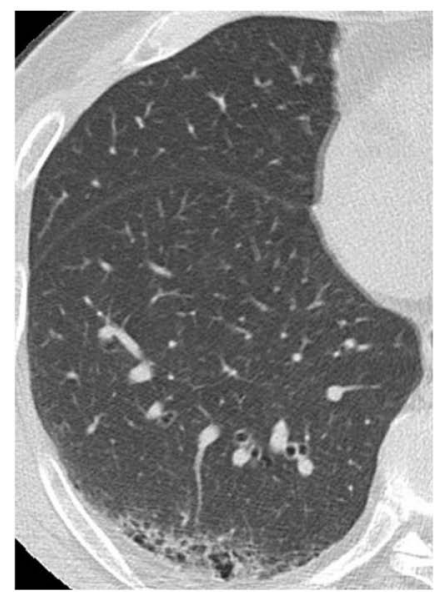

(a)

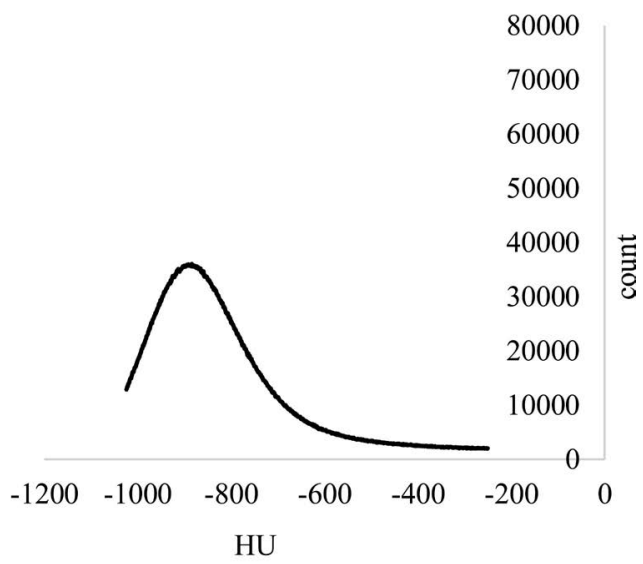

(b)

Figure 1. A 59-year-old man with interstitial lung abnormality. (a) Computed tomography $(\mathrm{CT})$ of the right lower lung zone. There was $80 \%$ normal attenuation (grading scale 1), $10 \%$ ground glass attenuation (grading scale 2 ), and $10 \%$ reticular abnormality (grading scale 3 ). The CT visual score of the right lower zone was calculated by multiplying each percentage area by each grading scale score: $80 \times 1+10 \times 2+10 \times 3=130$. The total score was the average of the upper, middle, and lower zone scores of both lungs; the CT visual score of this patient was 118. (b) Frequency histogram of this patient. Kurtosis was -0.992 and skewness was 0.743 .

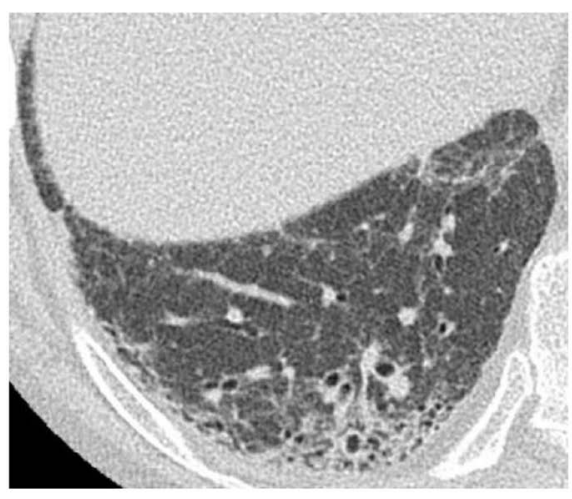

(a)

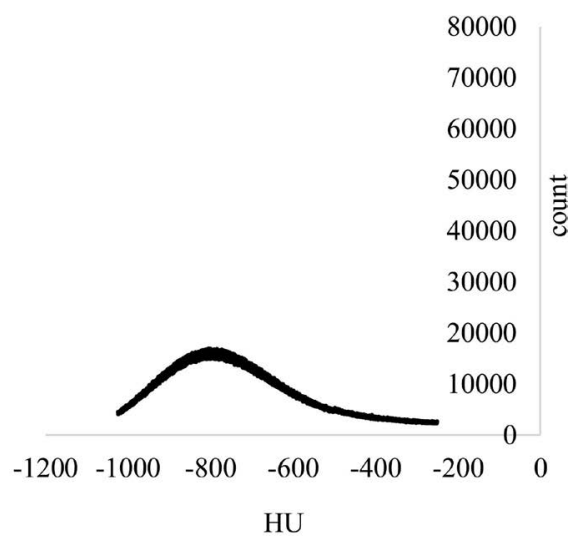

(b)

Figure 2. A 66-year-old woman with severe interstitial lung abnormality. (a) Computed tomography (CT) of the right lower lung zone. There was 50\% normal attenuation, $20 \%$ ground glass attenuation, $20 \%$ reticular abnormality, and $10 \%$ traction bronchiectasis. The CT visual score of the right lower zone was 130 and the total score was 168 . (b) The frequency histogram of this patient was less kurtotic and skewed than that of the patient in Figure 1. Kurtosis was -1.358 and skewness was 0.312 . 
$(-1024$ to $-250 \mathrm{HU}$ ) was created from the data remaining (see examples in Figure 1(b), Figure 2(b)). The kurtosis and skewness of the whole of both lungs were calculated as quantitative CT indexes of ILA.

\subsection{Statistical Analysis}

A chi-squared test was used to determine differences in sex, smoking status, and pathological stage among the three pathological groups. The Kruskal-Wallis test was used to determine differences in age among the three pathological groups. An intraclass correlation coefficient was used to determine the relationships between the CT visual scores assigned independently by the two radiologists. Spearman's rank correlation coefficient was used to determine the relationship between CT visual score and quantitative CT indexes. Spearman's rank correlation coefficient was also used to determine the relationships between severity of ILA and pathological stage. One-way analysis of variance was used to determine differences in the visual score and quantitative indexes among the three groups. A $P$-value less than 0.05 was considered statistically significant. All data were analyzed by using Statistical Software (IBM SPSS Statistics 21; IBM, Armonk, NY and Statcel 4; OMS, Tokyo, Japan).

\section{Results}

\subsection{Subjects' Characteristics and Pathological Stage of Concurrent Lung Cancer}

The clinical findings of the 28 patients are summarized in Table 1 . There were significant differences in age among the three groups $(P<0.03)$. There were no significant differences among the three groups in terms of gender, smoking status, or pathological stage. The pathological stage was IA in 6, IB in 9, IIA in 3, IIB in 4 , IIIA in 4 , and IIIB in 2 . AC was the predominant histologic type $(\mathrm{n}=$

Table 1. Subject characteristics and pathological stages of lung cancer.

\begin{tabular}{|c|c|c|c|c|c|c|}
\hline & & All $(\mathrm{n}=28)$ & $\mathrm{AC}(\mathrm{n}=13)$ & $\operatorname{SCC}(n=10)$ & OT $(n=5)$ & $P$ \\
\hline \multirow{2}{*}{ Sex, n } & Male & 21 & 7 & 9 & 5 & \multirow{2}{*}{0.051} \\
\hline & Female & 7 & 6 & 1 & 0 & \\
\hline Age, years, mean & & 71 & 75 & 69 & 66 & 0.03 \\
\hline \multirow{2}{*}{ Smoking status, No. } & smoker & 24 & 9 & 10 & 5 & \multirow{2}{*}{0.067} \\
\hline & Non-smoker & 4 & 4 & 0 & 0 & \\
\hline \multirow{6}{*}{ Pathological stage, No. } & IA & 6 & 2 & 4 & 0 & \multirow{6}{*}{0.36} \\
\hline & IB & 9 & 4 & 3 & 2 & \\
\hline & IIA & 3 & 1 & 2 & 0 & \\
\hline & IIB & 4 & 3 & 0 & 1 & \\
\hline & IIIA & 4 & 2 & 0 & 2 & \\
\hline & IIIB & 2 & 1 & 1 & 0 & \\
\hline
\end{tabular}

SCC: squamous cell carcinoma; AC: adenocarcinoma; OT: other. 
$13,46 \%)$ and SCC was the second most common $(\mathrm{n}=10,36 \%)$. OT consisted of 2 small-cell carcinoma, 1 large-cell carcinoma, 1 adenosquamous carcinoma, and 1 large-cell neuroendocrine carcinoma.

\subsection{CT Visual Scores and Quantitative Indexes}

The CT visual scores and quantitative indexes and their relationships are shown in Table 2. We investigated the inter-evaluator correlation of visual scores to confirm the score validity. Substantial reproducibility $(0.71)$ was confirmed between the CT visual scores assigned by the two independent observers. Therefore, we used the averages of the two observers' scores to evaluate the relationships with the quantitative indexes, the pathological stage and the histologic type. There was a significant negative correlation between visual score and skewness $(r=-0.39, P$ $=0.025)$, but kurtosis was not correlated with visual score $(r=-0.18, P=0.36)$. There was a significant positive correlation between kurtosis and skewness $(r$ $=0.94, P<0.0001)$ (Table 2$)$.

\subsection{Correlation between Severity of ILA and Pathological Stage}

There was a significant negative correlation between visual score and pathological stage $(r=-0.43, P=0.025)$. Neither kurtosis $(r=0.103, P=0.59)$ nor skewness $(r=0.122, P=0.53)$ was correlated with pathological stage (Table 2$)$.

\subsection{Correlation between Histologic Type and Severity of ILA}

Differences in quantitative CT indexes and CT visual score among the three pathological groups are shown in Table 3. There were no significant differences in the severity of ILA among the three pathological groups. Kurtosis in SCC was lower than in the other two groups, but the differences were not significant $(P=$ $0.26)$.

Table 2. Correlation coefficients ( $r$ values) between CT visual score, quantitative CT indexes, and pathological stage.

\begin{tabular}{ccccc}
\hline Parameter & CT visual score & Kurtosis & Skewness & Pathological stage \\
\hline CT visual score & 1 & & & \\
Kurtosis & -0.18 & 1 & & \\
Skewness & $-0.39(P=0.025)$ & $0.94(P<0.0001)$ & 1 & 1 \\
Pathological stage & $-0.43(P=0.025)$ & 0.10 & 0.12 & 1 \\
\hline
\end{tabular}

Table 3. CT visual scores and quantitative CT indexes in the three pathological groups.

\begin{tabular}{cccccc}
\hline & All $(\mathrm{n}=28)$ & AC $(\mathrm{n}=13)$ & SCC $(\mathrm{n}=10)$ & OT $(\mathrm{n}=5)$ & $P$ \\
\hline Visual score & $145.6 \pm 23.4$ & $144.6 \pm 23.5$ & $149.8 \pm 25.1$ & $140 \pm 23.2$ & 0.75 \\
Kurtosis & $-0.72 \pm 0.29$ & $-0.66 \pm 0.31$ & $-0.84 \pm 0.23$ & $-0.63 \pm 0.35$ & 0.26 \\
Skewness & $0.9 \pm 0.18$ & $0.91 \pm 0.21$ & $0.84 \pm 0.12$ & $0.96 \pm 0.21$ & 0.44
\end{tabular}

Data are means \pm SD. SCC: squamous cell carcinoma; AC: adenocarcinoma; OT: other. 


\section{Discussion}

To the best of our knowledge, this is the first paper to investigate the relationship between severity of ILA and stage of concurrent lung cancer. We found a negative correlation between visual score and pathological stage. This suggests that it is possible for patients to have only mild ILA but advanced lung cancer. One possible reason for this result is that patients with severe ILD may have undergone frequent examination; therefore, early-stage lung cancer may have been more likely to be detected in these patients. Although Best et al. [9] reported that quantitative CT indexes were correlated with the extent of ILD, in our histogram-based quantitative analysis there was no relationship between the quantitative indexes and the stage of concurrent lung cancer. Thus there was no relationship between the severity of ILA and the stage of concurrent lung cancer in the histogram analysis. Therefore, the frequency of follow-up examination should not be based on the severity of ILA in terms of either visual or histogram-based analysis on CT.

We found that kurtosis was lower in SCC than in the other two groups, but the differences were not significant. AC was the predominant histologic type and SCC was second. Some studies report that SCC is the most common histologic type in patients with idiopathic pulmonary fibrosis (IPF) [3] [4] [5] [15], whereas others show a predominance of AC [16] [17]. In Japan, AC is the predominant histologic type (58\% to $68 \%$ ), followed by SCC ( $21 \%$ to $22 \%)$ in all patients with lung cancer [18] [19]. The proportion of SCC in our study (i.e. 36\%) was higher than this. The results of Kawasaki et al. [20] suggest that the incidence of SCC in IPF patients (46\%) is significantly higher than that in non-IPF patents (22\%). Kawasaki et al. also suggested that $p 53$ gene alterations in squamous metaplasia might provide a clue to the molecular mechanisms underlying the high incidence of peripheral-type SCC in IPF patients [21].

There was a significant negative correlation between visual score and skewness, but kurtosis was not correlated with visual score. One possible reason for this lack of correlation is that honeycombing, which is the most severe CT visual score finding, forms low-attenuation areas on CT images, making kurtosis high.

Our study had several limitations. It was retrospective and involved only surgical cases. Furthermore, we selected only those patients who had undergone thin-section CT of the whole lung for automatic analysis by an image analysis system, so some patients were excluded from the study. In our study, ILA was diagnosed only from the CT findings, and there is no detailed pathological classification for ILDs. However, the diagnostics of ILD are still under discussion [22]. We agree that image-driven indexes could help in the evaluation of ILD severity [7] [8] [9]. Note that here we used the term "ILA" instead of "ILD" because there is as yet no confirmation of the concordance of CT and pathological findings in ILD.

\section{Conclusion}

It is possible for patients to have only mild ILA but advanced lung cancer. 
Therefore, the frequency of follow-up examination should not be based on the severity of ILA on CT.

\section{Conflicts of Interest}

None.

\section{References}

[1] Haddad, R. and Massaro, D. (1968) Idiopathic Diffuse Interstitial Pulmonary Fibrosis (Fibrosing Alveolitis), Atypical Epithelial Proliferation and Lung Cancer. The American Journal of Medicine, 45, 211-219. https://doi.org/10.1016/0002-9343(68)90039-9

[2] Fox, B. and Risdon, R. (1968) Carcinoma of the Lung and Diffuse Interstitial Pulmonary Fibrosis. Journal of Clinical Pathology, 21, 486-491. https://doi.org/10.1136/jcp.21.4.486

[3] Turner-Warwick, M., Lebowitz, M., Burrows, B. and Johnson, A. (1980) Cryptogenic Fibrosing Alveolitis and Lung Cancer. Thorax, 35, 496-469. https://doi.org/10.1136/thx.35.7.496

[4] Lee, H., Im, J., Ahn, J. and Yeon, K. (1996) Lung Cancer in Patients with Idiopathic Pulmonary Fibrosis: CT Findings. Journal of Computer Assisted Tomography, 20, 979-982. https://doi.org/10.1097/00004728-199611000-00020

[5] Sakai, S., Ono, M., Nishio, T., Kawarada, Y., Nagashima, A. and Toyoshima, S. (2003) Lung Cancer Associated with Diffuse Pulmonary Fibrosis: CT-Pathologic Correlation. Journal of Thoracic Imaging, 18, 67-71. https://doi.org/10.1097/00005382-200304000-00002

[6] Kishi, K., Homma, S., Kurosaki, A., Motoi, N. and Yoshimura, K. (2006) High-Resolution Computed Tomography Findings of Lung Cancer Associated with Idiopathic Pulmonary Fibrosis. Journal of Computer Assisted Tomography, 30, 95-99. https://doi.org/10.1097/01.rct.0000188650.66527.d2

[7] Best, A.C., Lynch, A.M., Bozic, C.M., Miller, D., Grunwald, G.K. and Lynch, D.A. (2003) Quantitative CT Indexes in Idiopathic Pulmonary Fibrosis: Relationship with Physiologic Impairment. Radiology, 228, 407-414.

https://doi.org/10.1148/radiol.2282020274

[8] Koyama, H., Ohno, Y., Yamazaki, Y., Nogami, M., Kusaka, A., Murase, K. and Sugimura, K. (2010) Quantitatively Assessed CT Imaging Measures of Pulmonary Interstitial Pneumonia: Effects of Reconstruction Algorithms on Histogram Parameters. European Journal of Radiology, 74, 142-146. https://doi.org/10.1016/j.ejrad.2009.02.006

[9] Best, A.C., Meng, J., Lynch, A.M., Bozic, C.M., Miller, D., Grunwald, G.K. and Lynch, D.A. (2008) Idiopathic Pulmonary Fibrosis: Physiologic Tests, Quantitative CT Indexes, and CT Visual Scores as Predictors of Mortality. Radiology, 246, 935-940. https://doi.org/10.1148/radiol.2463062200

[10] Oda, K., Ishimoto, H., Yatera, K., Naito, K., Ogoshi, T., Yamasaki, K., Imanaga, T., Tsuda, T., Nakao, H., Kawanami, T. and Mukae, H. (2014) High-Resolution CT Scoring System-Based Grading Scale Predicts the Clinical Outcomes in Patients with Idiopathic Pulmonary Fibrosis. Respiratory Research, 15, 10. https://doi.org/10.1186/1465-9921-15-10

[11] Raghu, G., Collard, H.R., Egan, J.J., Martinez, F.J., Behr, J., Brown, K.K., Colby, T.V., Cordier, J.-F.F., Flaherty, K.R., Lasky, J.A., Lynch, D.A., Ryu, J.H., Swigris, J.J., 
Wells, A.U., Ancochea, J., Bouros, D., Carvalho, C., Costabel, U., Ebina, M., Hansell, D.M., Johkoh, T., Kim, D.S., King, T.E., Kondoh, Y., Myers, J., Müller, N.L., Nicholson, A.G., Richeldi, L., Selman, M., Dudden, R.F., Griss, B.S., Protzko, S.L. and Schünemann, H.J. (2011) An Official ATS/ERS/JRS/ALAT Statement: Idiopathic Pulmonary Fibrosis: Evidence-Based Guidelines for Diagnosis and Management. American Journal of Respiratory and Critical Care Medicine, 183, 788-824. https://doi.org/10.1164/rccm.2009-040GL

[12] Travis, W.D., Costabel, U., Hansell, D.M., King, T.E., Lynch, D.A., Nicholson, A.G., Ryerson, C.J., Ryu, J.H., Selman, M., Wells, A.U., Behr, J., Bouros, D., Brown, T.V., Colby, H.R., Collard, C.R., Cordeiro, V., Cottin, B., Crestani, M., Drent, R.F., Dudden, J., Egan, K., Flaherty, C., Hogaboam, Y., Inoue, K.K., Johkoh, T., Kim, D.S., Kitaichi, M., Loyd, J., Martinez, F.J., Myers, J., Protzko, S., Raghu, G., Richeldi, L., Sverzellati, N., Swigris, J. and Valeyre, D. (2013) An Official American Thoracic Society/European Respiratory Society Statement: Update of the International Multidisciplinary Classification of the Idiopathic Interstitial Pneumonias. American Journal of Respiratory and Critical Care Medicine, 188, 733-748. https://doi.org/10.1164/rccm.201308-1483ST

[13] Colice, G.L., Shafazand, S., Griffin, J.P., Keenan, R. and Bolliger, C.T. (2007) Physiologic Evaluation of the Patient with Lung Cancer Being Considered for Resectional Surgery: ACCP Evidenced-Based Clinical Practice Guidelines (2nd Edition). Chest, 132, 161S-177S. https://doi.org/10.1378/chest.07-1359

[14] Ichikado, K., Suga, M., Muranaka, H., Gushima, Y., Miyakawa, H., Tsubamoto, M., Johkoh, T., Hirata, N., Yoshinaga, T., Kinoshita, Y., Yamashita, Y. and Sasaki, Y. (2006) Prediction of Prognosis for Acute Respiratory Distress Syndrome with Thin-Section CT: Validation in 44 Cases. Radiology, 238, 321-329. https://doi.org/10.1148/radiol.2373041515

[15] Aubry, M.C., Myers, J.L., Douglas, W.W., Tazelaar, H.D., Washington Stephens, T.L., Hartman, T.E., Deschamps, C. and Pankratz, V.S. (2002) Primary Pulmonary Carcinoma in Patients with Idiopathic Pulmonary Fibrosis. Mayo Clinic Proceedings, 77, 763-770. https://doi.org/10.4065/77.8.763

[16] Kawai, T., Yakumaru, K., Suzuki, M. and Kageyama, K. (1987) Diffuse Interstitial Pulmonary Fibrosis and Lung Cancer. Acta Pathologica Japonica, 37, 11-19. https://doi.org/10.1111/j.1440-1827.1987.tb03130.x

[17] Matsushita, H., Tanaka, S., Saiki, Y., Hara, M., Nakata, K., Tanimura, S. and Banba, J. (1995) Lung Cancer Associated with Usual Interstitial Pneumonia. Pathology International, 45, 925-932. https://doi.org/10.1111/j.1440-1827.1995.tb03417.x

[18] Nakaya, N., Goto, K., Saito-Nakaya, K., Inagaki, M., Otani, T., Akechi, T., Nagai, K., Hojo, F., Uchitomi, Y., Tsugane, S. and Nishiwaki, Y. (2006) The Lung Cancer Database Project at the National Cancer Center, Japan: Study Design, Corresponding Rate and Profiles of Cohort. Japanese Journal of Clinical Oncology, 36, 280-284. https://doi.org/10.1093/jico/hyl015

[19] Sawabata, N., Fujii, Y., Asamura, H., Nomori, H., Nakanishi, Y., Eguchi, K., Mori, M., Okumura, M., Miyaoka, E. and Yokoi, K. (2011) Lung Cancer in Japan: Analysis of Lung Cancer Registry Cases Resected in 2004. The Journal of the Japanese Respiratory Society, 49, 327-342. https://doi.org/10.2482/haigan.50.875

[20] Kawasaki, H., Nagai, K., Yokose, T., Yoshida, J., Nishimura, M., Takahashi, K., Suzuki, K., Kakinuma, R. and Nishiwaki, Y. (2001) Clinicopathological Characteristics of Surgically Resected Lung Cancer Associated with Idiopathic Pulmonary Fibrosis. Journal of Surgical Oncology, 76, 53-57.

https://doi.org/10.1002/1096-9098(200101)76:1<53::AID-JSO1009>3.0.CO;2-T 
[21] Kawasaki, H., Ogura, T., Yokose, T., Nagai, K., Nishiwaki, Y. and Esumi, H. (2001) p53 Gene Alteration in Atypical Epithelial Lesions and Carcinoma in Patients with Idiopathic Pulmonary Fibrosis. Human Pathology, 32, 1043-1049.

https://doi.org/10.1053/hupa.2001.28246

[22] Lynch, D.A., Sverzellati, N., Travis, W.D., Brown, K.K., Colby, T.V., Galvin, J.R., Goldin, J.G., Hansell, D.M., Inoue, Y., Johkoh, T., Nicholson, A.G., Knight, S.L., Raoof, S., Richeldi, L., Ryerson, C.J., Ryu, J.H. and Wells, A.U. (2018) Diagnostic Criteria for Idiopathic Pulmonary Fibrosis: A Fleischner Society White Paper. The Lancet Respiratory Medicine, 6, 138-153.

https://doi.org/10.1016/S2213-2600(17)30433-2 